\title{
Maternal weight and excessive weight gain during pregnancy modify the immunomodulatory potential of breast milk
}

\author{
Maria Carmen Collado ${ }^{1,2}$, Kirsi Laitinen ${ }^{2,3}$, Seppo Salminen ${ }^{2,3}$ and Erika Isolauri ${ }^{4}$
}

INTRODUCTION: Breast milk is an optimal source of nutrition for infants. It contains bioactive components including bacteria that support the microbial colonization and immune system development of the infant. The determinants of human milk composition remain poorly understood, although maternal nutritional and immunological status as well as lifestyle and dietary habits seem to have an impact.

METHODS: The subjects selected were women from a prospective follow-up study categorized by BMI. Milk samples were taken after delivery and at 1 and 6 mo later for analysis of composition in regard to transforming growth factor (TGF)- $\beta 2$, soluble CD14 (sCD14), cytokines, and microbiota.

RESULTS: TGF- $\beta 2$ and sCD14 levels in the breast milk of overweight mothers tended to be lower than the levels in that of normal-weight mothers. Also, higher levels of Staphylococcus group bacteria and lower levels of Bifidobacterium group bacteria were detected in overweight mothers as compared with normal-weight ones. The prevalence of Akkermansia muciniphila-type bacteria was also higher in overweight mothers, and the numbers of these bacteria were related to the interleukin (IL)-6 concentration in the colostrum, which was in turn related to lower counts of Bifidobacterium group bacteria in the breast milk of overweight women.

DISCUSSION: Complex interactions of cytokines and microbiota in breast milk guide the microbiological, immunological, and metabolic programming of infant health. Our data may indicate the presence of an additional mechanism that may explain the heightened risk of obesity for infants of overweight and excessive weight gain mothers.

$T$ he complex and distinct composition of human milk may exert specific effects on infant nutrition, immunological competence, and later health $(1,2)$. Protection may be provided by components including regulatory cytokines and growth factors as well as by other compounds such as peptides, certain fatty acids, and oligosaccharides. These latter support innate immunity and guide the development of adaptive immunity, their importance being at a peak during the first months of lactation when breast milk is the sole source of nutrition for the infant. Breast milk is also a continuous source of microbes, their growth factors, and components that regulate host-microbe interactions. This underscores the key role of breast feeding in conferring immunological protection during a vulnerable period in life, when the infant's own immune defenses, including the integrity of the gut barrier, are immature (3-5). The determinants of human milk composition remain poorly understood, although the mother's lifestyle, nutritional and immunological status, and dietary habits likely explain the variations $(6,7)$.

Developed countries are experiencing a progressive increase in immunological and metabolic diseases, and the velocity of spread of these is particularly conspicuous in children. Deviations from the microbiota characteristic of the healthy breast-fed infant are associated with a heightened risk of allergic and inflammatory conditions and, as recently reported, obesity $(8,9)$. In fact, recent advances in experimental studies have shed light on both the underlying mechanism through which the gut microbiota regulate the harvesting and storage of energy $(10,11)$ and the mechanism through which systemic low-grade inflammation results in obesity (12). In the case of pregnant overweight women, a vicious cycle of unfavorable metabolic development may be generated if the aberrations in gut microbiota patterns related to overweight or excessive weight gain during pregnancy are transferred to the infant (13). We have hypothesized here that these interactions may take place beyond the gastrointestinal tract and after birth; the key bioactive components in breast milk, namely, the cytokines and microbiota, may depend on maternal weight and BMI and also on weight gain during pregnancy. To test this hypothesis, we carried out a longitudinal study of milk samples in order to analyze the relationship between cytokines and microbiota and to explore the maternal influences on these.

\section{RESULTS}

\section{Clinical Characteristics}

The clinical characteristics of mother-infant pairs $(n=56)$ are shown in Table 1. Infants of overweight subjects had significantly

\footnotetext{
'Department of Biotechnology, Institute of Agrochemistry and Food Science, Spanish National Research Council (IATA-CSIC), Valencia, Spain; ${ }^{2}$ Functional Foods Forum, University of Turku, Turku, Finland; ${ }^{3}$ Institute of Biomedicine, University of Turku, Turku, Finland; ${ }^{4}$ Department of Pediatrics, Turku University Hospital and University of Turku, Turku, Finland. Correspondence: Maria Carmen Collado (mcolam@iata.csic.es)
} 
Table 1. Clinical characteristics of all women included in the study and characteristics according to pregestational BMI group (BMI $\leq 25$ and BMI >25) before delivery

\begin{tabular}{|c|c|c|c|c|}
\hline & Total $(n=56)$ & $\mathrm{BMI} \leq 25(n=34)$ & $\mathrm{BMI}>25(n=22)$ & $P$ value \\
\hline \multicolumn{5}{|l|}{ Mothers } \\
\hline Age (y) & $30.23(4.90)$ & $29.40(26.25-33.38)$ & $29.96(26.61-34.36)$ & 0.990 \\
\hline Height before pregnancy (cm) & $167.5(5.14)$ & $168.0(163.7-171.0)$ & $166.3(163.4-172.7)$ & 0.733 \\
\hline Weight before pregnancy (kg) & $72.44(15.30)$ & $59.5(57.40-62.25)$ & $89.0(82.75-92.75)$ & 0.000 \\
\hline BMI before pregnancy & $21.78(5.38)$ & $21.64(20.87-23.10)$ & $31.70(29.34-32.93)$ & 0.000 \\
\hline Weight gain over pregnancy (kg) & $14.50(4.70)$ & $15.00(11.20-17.60)$ & $12.5(10.25-17.93)$ & 0.261 \\
\hline Mode of delivery (\% vaginal) & $43 / 56(76.8 \%)$ & $28 / 34(88.3 \%)$ & $15 / 22(68.2 \%)$ & 0.220 \\
\hline Duration of gestation (wk) & $40.26(1.14)$ & 40.30 (39.54-41.14) & $40.43(39.14-41.29)$ & 0.700 \\
\hline Primipara (\%) & $33 / 56(59.0 \%)$ & $21 / 34(61.8 \%)$ & $12 / 22(54.5 \%)$ & 0.592 \\
\hline Exclusive breastfeeding period (mo) & $3.00(2.03)$ & $3.00(0.75-4.38)$ & $4.00(0.50-5.00)$ & 0.378 \\
\hline Breastfeeding at infant 1 mo old (\%) & $53 / 56(94.6 \%)$ & $34 / 34(100 \%)$ & 19/22 (86.4\%) & 0.027 \\
\hline Breastfeeding at infant 6 mo old (\%) & $37 / 56(66.1 \%)$ & $21 / 34(61.8 \%)$ & $16 / 22(72.7 \%)$ & 0.397 \\
\hline \multicolumn{5}{|l|}{ Infants } \\
\hline Infant gender (\% female) & $30 / 56(53.6 \%)$ & $18 / 34(52.9 \%)$ & $12 / 22(54.5 \%)$ & 0.906 \\
\hline Head circumference at birth (cm) & $35.2(1.35)$ & $35.0(34.0-36.0)$ & $35.5(34.6-36.0)$ & 0.177 \\
\hline \multicolumn{5}{|l|}{ Weight (kg) } \\
\hline Birth & $3.61(0.42)$ & $3.52(3.33-3.75)$ & $3.81(3.36-4.13)$ & 0.030 \\
\hline 6 mo old & $8.02(1.02)$ & $7.92(7.32-8.73)$ & $8.54(7.76-8.90)$ & 0.209 \\
\hline \multicolumn{5}{|l|}{ Height (cm) } \\
\hline Birth & $51.40(1.85)$ & $51.0(50.0-52.0)$ & $52.0(50.0-53.7)$ & 0.334 \\
\hline $6 \mathrm{mo}$ old & $68.15(2.80)$ & $68.00(67.2-70.0)$ & $69.0(67.7-71.00)$ & 0.236 \\
\hline \multicolumn{5}{|l|}{ Infant diet at 1 mo old (\%) } \\
\hline Exclusive breastfeeding & $38 / 56(67.8 \%)$ & $23 / 34(67.6 \%)$ & $15 / 22(68.2 \%)$ & 0.967 \\
\hline Partial breastfeeding & $15 / 56(26.8 \%)$ & $11 / 34(32.3 \%)$ & $4 / 22(18.2 \%)$ & 0.242 \\
\hline Nonbreastfeeding & $3 / 56(5.4 \%)$ & $0 / 34(0 \%)$ & $3 / 22(13.6 \%)$ & 0.027 \\
\hline \multicolumn{5}{|l|}{ Infant diet at 6 mo old (\%) } \\
\hline Exclusive breastfeeding & $0 / 56(0 \%)$ & $0 / 34(0 \%)$ & $0 / 22(0 \%)$ & - \\
\hline Breast milk + solid food & $36 / 56(64.3 \%)$ & $21 / 34(61.8 \%)$ & $15 / 22(68.2 \%)$ & 0.625 \\
\hline Nonbreastfeeding & $20 / 56(35.7 \%)$ & $13 / 34$ (38.2\%) & $7 / 22(31.8 \%)$ & 0.227 \\
\hline
\end{tabular}

Data for all mothers included are shown as average and SD or percentages (\%). Data for BMI comparison are shown as median and interquartile range (IQR) or percentages (\%) and statistical differences were calculated using Mann-Whitney $U$-test and $X^{2}$-square test, respectively. Infant characteristics for each group are included.

higher birth weights as compared with those of normal-weight subjects. All the mothers commenced breastfeeding their infants after delivery; $94.6 \%$ continued up to 1 mo and $66.1 \%$ continued up to $6 \mathrm{mo}$ (Table 1). However, the mean duration of exclusive breastfeeding was 3.00 (SD 2.03) mo. None of the subjects required antibiotic treatment before delivery, although some of them received antibiotics during delivery $(8 / 56,14.3 \%)$ and after delivery $(6 / 56,10.70 \%)$ because of the presence of group B Streptococcus or for standard coverage for Cesarean sections. Some of the subjects developed gestational diabetes during pregnancy $(18 / 56,32.1 \%)$. All these cases were distributed equally among the groups, and no differences between groups were found. Other infections and problems were not reported.

Cytokine and Microbiota Composition in Breast Milk During the First Months of Lactation

The colostrum samples showed higher concentrations of transforming growth factor $(\mathrm{TGF}-\beta)(P=0.004)$, soluble CD14
(sCD14) $(P=0.0001)$, and interleukin (IL)-6 $(P=0.0001)$ and lower concentrations of interferon $(\operatorname{IFN})-\gamma(P=0.022)$ than the 1-mo milk samples (Table 2).

A comparison of the microbial counts (log-scale) in colostrum (within 24-48 h after delivery) and mature milk (at 1 and 6 mo after delivery) (Table 3 ) showed significantly higher counts of the Enterococcus group in colostrum than in the milk samples taken at $1 \mathrm{mo}$ after delivery $(P=0.011)$, with a further decrease being observed in the mature milk samples $(P=0.045)$. The opposite was seen for the Staphylococcus aureus and Clostridium coccoides group counts, which were lower in colostrum than in mature milk samples $(P=0.036$ and $P=0.0005$, respectively).

\section{Interaction Between Breast Milk Cytokines and the Composition of Microbiota}

The presence of Bifidobacterium-group bacteria was negatively associated with concentrations of IFN- $\gamma(P=0.030)$, whereas 
Table 2. Cytokine levels in breast milk samples and changes (ratio) in levels from colostrum to 1-mo samples

\begin{tabular}{|c|c|c|c|c|c|c|c|}
\hline \multirow[b]{3}{*}{ Cytokine level } & \multicolumn{4}{|c|}{ Breast milk sample } & & & \multirow[b]{3}{*}{$P$ value $^{b}$} \\
\hline & \multicolumn{2}{|c|}{ Colostrum $(n=43)^{\mathrm{a}}$} & \multicolumn{2}{|c|}{$1 \mathrm{mo}(n=44)^{\mathrm{a}}$} & \multicolumn{2}{|c|}{ Ratio colostrum/1 mo } & \\
\hline & Mean & $95 \% \mathrm{Cl}$ & Mean & $95 \% \mathrm{Cl}$ & Mean & $95 \% \mathrm{Cl}$ & \\
\hline $\mathrm{sCD} 14(\mu \mathrm{g} / \mathrm{ml})$ & 26.23 & $21.99-31.28$ & 5.08 & $4.22-6.11$ & 0.19 & $0.15-0.24$ & 0.0001 \\
\hline $\mathrm{IFN}-\gamma(\mathrm{pg} / \mathrm{ml})$ & 129.18 & $100.84-165.50$ & 196.57 & $152.75-252.95$ & 1.52 & $1.06-2.16$ & 0.022 \\
\hline IL10 (pg/ml) & 9.63 & $7.47-12.40$ & 11.57 & $8.89-15.06$ & 1.20 & $0.83-1.72$ & 0.299 \\
\hline IL6 (pg/ml) & 69.07 & $51.23-93.14$ & 18.63 & $13.74-25.27$ & 0.28 & $0.17-0.41$ & 0.0001 \\
\hline IL4 (pg/ml) & 19.12 & $14.96-24.46$ & 20.60 & $15.96-26.60$ & 1.07 & $0.75-1.54$ & 0.665 \\
\hline IL2 (pg/ml) & 29.38 & $23.22-37.17$ & 22.73 & $17.81-29.01$ & 0.77 & $0.56-1.05$ & 0.100 \\
\hline
\end{tabular}

$\mathrm{Cl}$, confidence interval; IFN, interferon; IL, interleukin; SCD14, soluble CD14;TGF, transforming growth factor; TNF, tumor necrosis factor.

aData are shown as mean of bioactive factor levels and $95 \% \mathrm{Cl}$. bStatistical differences were calculated using mixed models from colostrum to 1 mo of exclusive breastfeeding. A P value $<0.05$ was considered statistically significant.

the Streptococcus-group bacteria were positively related to concentrations of IFN- $\gamma(P=0.001)$, tumor necrosis factor (TNF)- $\alpha$ $(P=0.020)$, and IL10 $(P=0.001)$. In addition, proinflammatory cytokine IL6 was linked to higher levels of Staphylococcus group $(P=0.03)$ in colostrum, whereas anti-inflammatory IL10 and IL4 were associated with lower levels of Akkermansia muciniphila ( $P=0.050$ and $P=0.055$, respectively).

Impact of Maternal BMI and Weight Gain During Pregnancy on Cytokine Concentrations in Breast Milk

No significant differences in cytokine concentrations were observed in the breast milk samples of overweight subjects as compared with those of normal-weight subjects (Table 4). There were also no cytokine differences in the samples of subjects who gained excessive weight during pregnancy as compared with those of subjects who had normal weight gain (data not shown). However, relationships were found between cytokines and maternal weight, BMI, and weight gain. Lower levels of TGF- $\beta 2(r=-0.629, P=0.009)$, sCD14 $(r=-0.444$, $P=0.020)$, and IL6 $(r=-0.600, P=0.001)$ were related to higher BMI and weight after 1 mo of breastfeeding.

Impact of Maternal BMI and Weight Gain on the Composition of Breast Milk Microbiota

Higher counts of Staphylococcus-group bacteria and lower counts of Bifidobacterium-group bacteria were detected in overweight subjects in both 1 -mo $(P=0.023$ and $P=0.009$, respectively) and 6-mo milk samples ( $P=0.023$ and $P=0.040$, respectively) than in normal-weight subjects (Table 5). In addition, Staphylococcus-group bacteria were more prevalent $(P=0.043$ in colostrum and $P=0.036$ at $1 \mathrm{mo})$ in milk samples from overweight subjects than in those from normal-weight subjects. A similar tendency was observed with respect to Staphylococcus aureus-group bacteria, but this was significant only in the 6-mo samples $(P=0.017)$. Although the counts of Bifidobacterium-group bacteria were higher in samples from normal-weight subjects than in those from overweight subjects, the difference did not reach statistical significance either in colostrum $(P=0.054)$ or in the 1 -mo samples $(P=0.068)$.
Using a mixed-models approach to analyze the effect of BMI on the composition of breast milk microbiota during lactation, we found that, over the first 6 mo of breastfeeding, overweight subjects had higher total bacteria counts (ratio $=0.34$, $P=0.011,95 \%$ confidence interval (CI) $0.08-0.60$ ), higher counts of the Staphylococcus group (ratio $=0.62, P=0.0001$, 95\% CI $0.30-0.93$ ) and the Lactobacillus group (ratio = $0.52, P=0.038,95 \%$ CI $0.02-2.02$ ), and lower counts of the Bifidobacterium group (ratio $=-0.48, P=0.002,95 \% \mathrm{CI}-0.78$ to -0.18) than normal-weight subjects. These results indicated that overweight subjects had 0.48 times fewer Bifidobacterium bacteria, 0.62 times more Staphylococcus bacteria, and 0.52 times more Lactobacillus group bacteria during lactation as compared with normal-weight subjects.

The quantum of weight gain during pregnancy had an impact on the composition of breast milk microbiota during the first 6 mo of breastfeeding (Table 6). Excessive weight gain during pregnancy was associated with higher levels of Staphylococcus group in colostrum $(P=0.050)$ and lower counts of Bifidobacterium group bacteria in 1-mo samples $(P=0.030)$. Those who gained excessive weight during pregnancy had 0.42 times fewer Bifidobacterium-group bacteria during lactation than normal-weight-gain subjects $\operatorname{did}(b=-0.42, P=0.004$, $95 \%$ CI -0.71 to -0.14$)$. No such differences were found with respect to other bacterial groups.

Effect of Maternal BMI and Pregnancy Weight Gain on the Interaction Between Breast Milk Cytokines and Microbiota Composition

Using a mixed-models approach to analyze relationships, it was found that, during the first month of breastfeeding, higher concentrations of sCD14 in normal-weight mothers were related to higher counts of Bifidobacterium-group bacteria (ratio $=1.18, P=0.024,95 \%$ CI 0.98-1.58). Higher concentrations of IL10 and IL4 were associated with lower counts of Akkermansia muciniphila (ratio $=0.76, P=0.010,95 \%$ CI 0.63 0.90 and ratio $=0.70, P=0.038,95 \%$ CI $0.50-0.97$, respectively). Furthermore, IL10 (ratio $=1.62, P=0.020,95 \%$ CI 1.08-2.42) and IFN- $\gamma($ ratio $=1.70, P=0.026,95 \%$ CI $1.06-2.70)$ were 


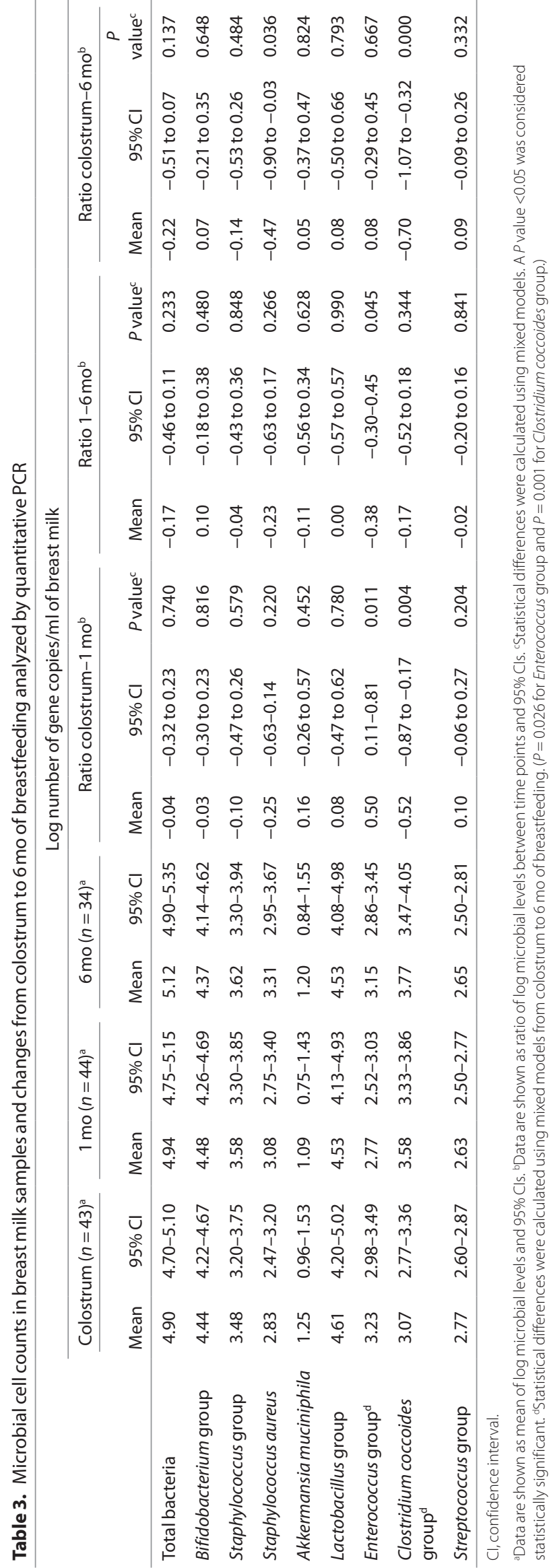

related to the levels of the Streptococcus group during lactation. Higher concentrations of IL6 in colostrum samples were related to higher counts of Staphylococcus ( $r=0.628, P=0.039)$ in normal-weight subjects, lower levels of Akkermansia muciniphila ( $r$ $=-0.738, P=0.015)$ in overweight subjects, and lower counts of Bifidobacterium-group bacteria $(r=-0.629, P=0.038)$ in subjects with excessive weight gain during pregnancy.

\section{DISCUSSION}

Breastfeeding is important for the health of the infant and is therefore recommended. It is well characterized that the nutritional and immunological composition of human milk changes over the lactation period (14) to meet the needs of the growing infant and contribute to the development of his/her host defense mechanisms, particularly in the gastrointestinal tract where the major load of new antigens is encountered. Our study is the first to reveal equal modification in the composition of breast milk microbiota. Further research is required to explore the impact of this finding, in view of the demonstrated interaction of microbiota composition with immunoregulatory sCD14 and TGF- $\beta 2$, as well as the pivotal importance of mother's weight and weight gain during pregnancy and during lactation.

A delicate balance of stimulatory, even proinflammatory, maturational signals, together with myriad anti-inflammatory compounds, is transferred from mother to infant via breastfeeding. Breast milk-derived secretory IgA contributes to the immune exclusion of antigens in infants (in whom endogenous intestinal IgA production is still immature) (5), thereby preventing detrimental inflammatory reactions to dietary antigens and nonpathogenic microbes. Of note, breastfeeding not only confers passive protection but also actively stimulates the development of the infant's own immune system, with TGF- $\beta$ regulating both IgA production (an indispensable component of the mucosal immune defense) and oral tolerance induction $(15,16)$. Endogenous TGF- $\beta$ production in the intestine is sparse during the first days of life and gradually increases thereafter (17), whereas the exogenous supply, i.e., TGF- $\beta$ in maternal milk, peaks immediately after birth in colostrum and decreases significantly in mature milk, suggesting a shift from an exogenous to an endogenous source of supply. These findings were also documented in our study. The same evolution pattern was seen in our study with respect to sCD14 and IL6. The latter are associated with reduction in risk of allergy, atopic dermatitis, asthma, and obesity $(18,19)$ and with indirect anti-inflammatory potential via stimulation of IgA synthesis $(16,20)$. The correlations between the concentrations of cytokines involved in IgA synthesis, i.e., IL-10, IL-6, and TGF- $\beta$, may explain the stimulatory effect on IgA production in breast-fed babies (20).

The clinical correlate appears to be reduced risk of allergic disease; high concentrations of TGF- $\beta$ in breast milk were associated with prevention of early atopic eczema in breastfed infants (21). The involvement of microbial provocation has only recently been suggested, with rigorous research in the field of healthy-gut microbiota showing that specific probiotics differently regulate the generation of TGF- $\beta(16,22)$ and 
Table 4. Cytokine concentrations and changes in breast milk according to maternal BMI

\begin{tabular}{|c|c|c|c|c|c|}
\hline & \multirow{2}{*}{$\begin{array}{c}\mathrm{BMI} \\
\leq 25 \mathrm{~kg} / \mathrm{m}^{2}\end{array}$} & \multirow{2}{*}{$\begin{array}{c}\mathrm{BMI} \\
>25 \mathrm{~kg} / \mathrm{m}^{2}\end{array}$} & \multicolumn{3}{|c|}{ Ratio $\mathrm{BMI} \leq 25$ to $\mathrm{BMI}>25$} \\
\hline & & & Mean & $95 \% \mathrm{Cl}$ & $P$ value \\
\hline \multicolumn{6}{|l|}{ TGF- $\beta$ (pg/ml) } \\
\hline Colostrum & $2,247.06$ & $1,300.67$ & 1.93 & $0.83-2.62$ & 0.094 \\
\hline $1 \mathrm{mo}$ & $1,006.03$ & 550.0 & 1.83 & $0.71-4.70$ & 0.188 \\
\hline Lactation & - & - & 1.88 & $0.95-3.34$ & 0.071 \\
\hline \multicolumn{6}{|l|}{$\mathrm{sCD} 14(\mu \mathrm{g} / \mathrm{ml})$} \\
\hline Colostrum & 28.22 & 23.21 & 1.22 & $0.84-1.75$ & 0.275 \\
\hline $1 \mathrm{mo}$ & 5.54 & 4.35 & 1.27 & $0.86-1.88$ & 0.210 \\
\hline Lactation & - & - & 1.24 & $0.92-1.67$ & 0.090 \\
\hline \multicolumn{6}{|l|}{$\mathrm{IFN}-\gamma(\mathrm{pg} / \mathrm{ml})$} \\
\hline Colostrum & 122.60 & 141.91 & 0.83 & $0.51-1.46$ & 0.566 \\
\hline $1 \mathrm{mo}$ & 201.57 & 187.0 & 1.08 & $0.62-1.86$ & 0.775 \\
\hline Lactation & - & - & 0.97 & $0.66-1.41$ & 0.845 \\
\hline \multicolumn{6}{|l|}{ TNF-a (pg/ml) } \\
\hline Colostrum & 9.87 & 11.41 & 0.86 & $0.54-1.38$ & 0.560 \\
\hline $1 \mathrm{mo}$ & 10.60 & 10.23 & 1.02 & $0.52-1.87$ & 0.770 \\
\hline Lactation & - & - & 0.97 & $0.68-1.30$ & 0.845 \\
\hline \multicolumn{6}{|l|}{ IL10 (pg/ml) } \\
\hline Colostrum & 8.80 & 11.35 & 0.77 & $0.45-1.32$ & 0.330 \\
\hline $1 \mathrm{mo}$ & 11.46 & 11.80 & 0.97 & $0.55-1.70$ & 0.915 \\
\hline Lactation & - & - & 0.87 & $0.58-1.30$ & 0.455 \\
\hline \multicolumn{6}{|l|}{ IL6 (pg/ml) ${ }^{\mathrm{a}}$} \\
\hline Colostrum & 62.86 & 81.85 & 0.77 & $0.41-1.42$ & 0.381 \\
\hline $1 \mathrm{mo}$ & 22.12 & 13.22 & 1.67 & $0.18-3.16$ & 0.107 \\
\hline Lactation & - & - & 1.13 & $0.72-1.76$ & 0.561 \\
\hline \multicolumn{6}{|l|}{ IL4 (pg/ml) } \\
\hline Colostrum & 18.23 & 20.80 & 0.88 & $0.64-1.38$ & 0.604 \\
\hline $1 \mathrm{mo}$ & 20.74 & 20.34 & 1.02 & $0.52-1.48$ & 0.941 \\
\hline Lactation & - & - & 0.94 & $0.65-1.40$ & 0.760 \\
\hline \multicolumn{6}{|l|}{ IL2 (pg/ml) } \\
\hline Colostrum & 29.30 & 29.40 & 1.00 & $0.60-1.64$ & 0.987 \\
\hline $1 \mathrm{mo}$ & 21.00 & 26.38 & 0.80 & $0.47-1.34$ & 0.370 \\
\hline Lactation & - & - & 0.90 & $0.60-1.31$ & 0.536 \\
\hline
\end{tabular}

The results for study groups are given as geometric means and the group comparison as ratio normal (BMI $\leq 25)$ to overweight $(\mathrm{BMI}>25)$ with $95 \% \mathrm{Cl}$. Colostrum samples: $\mathrm{BMI}$ $\leq 25 \mathrm{~kg} / \mathrm{m}^{2}(n=23)$ and BMI $>25(n=21) ; 1$-mo milk samples: $\mathrm{BMI} \leq 25 \mathrm{~kg} / \mathrm{m}^{2}(n=27)$ and $\mathrm{BMI}>25(n=17)$.

$\mathrm{Cl}$, confidence interval; IFN, interferon; IL, interleukin; SCD14, soluble CD14;TGF, transforming growth factor; TNF, tumor necrosis factor.

${ }^{\mathrm{B} B M l}$ and time effect $P$ value $<0.05$.

immunoglobulin response as specific IgA responses $(23,24)$ and have not been shown to interfere with the immune response to vaccines; they may also improve seroconversion rates $(25,26)$.

Breast milk has been to shown to be a source of microbes such as staphylococci, streptococci, lactic acid bacteria, and bifidobacteria (4). Bacterial translocation has been demonstrated in different parts of the body, including the gut $(27,28)$. Dendritic cells that penetrate the intestinal epithelium take up commensal bacteria from the gut lumen and reach the systemic circulation, retaining even live bacteria for days (29). More recently, their transfer to the mammary glands has been reported $(27,28,30)$. Bifidobacteria are the hallmark of the gut microbiota in healthy breast-fed infants, and, apart from bifidogenic oligosaccharides, specific and distinctive species of Bifidobacterium are present in breast milk (4). In our study, we found that higher counts of Bifidobacterium- and Staphylococcus-group bacteria in breast milk were associated with higher sCD14 concentrations, with the latter group of bacteria being also associated with higher concentrations of IL6. In contrast, Akkermansia muciniphila tended to be associated with proinflammatory signals, namely, higher TNF- $\alpha$ and IFN- $\gamma$ concentrations in colostrum and lower concentrations of IL10 and IL4 during lactation. Indeed, we were surprised to detect this mucin-degrading gut bacterium in breast milk, notwithstanding its previously reported presence among gut microbiota in infants (31). Given its capability to degrade intestinal mucus, Akkermansia muciniphila may indirectly affect the innate and adaptive immune responses (32). In light of the findings of a recent metagenomic study, however, the cascade of events linking Akkermansia muciniphila to the pathogenesis of inflammatory diseases may have been misinterpreted; although this microorganism contains numerous candidate mucinase-encoding genes, it lacks genes encoding canonical mucus-binding domains, and may thereby actually protect the mucus lining by controlling other mucosa degraders (33). Akkermansia muciniphila has not been correlated with any disease or sign of pathogenicity, and is an inhabitant of the gut microbial ecosystem $(32,33)$.

Our study extends the known host-microbe cross-talk in breast milk to the mother's weight and weight gain during pregnancy. High prepregnancy BMI and excessive weight gain during pregnancy have previously been associated with aberrations in the composition of microbiota in the maternal gut (34) which acts as the inoculum for the development of microbiota in the infant gut. Here again, breast milk is recognized as the single most important postpartum element in metabolic and immunological programming of the child's health (35). We detected lower TGF- $\beta$ and sCD14 in breast milk samples of overweight subjects as compared with those from normal-weight subjects. This finding may mirror a $\mathrm{T}$ helper 2-polarized responder type previously reported to be associated with the allergic state of the lactating mother, found from comparing colostrum samples from allergic mothers with those from nonallergic mothers (20). Correspondingly, the counts of Bifidobacterium-group bacteria were lower whereas those of Staphylococcus-group microbes and Akkermansia muciniphila levels were higher in milk samples of overweight mothers than in those of normal-weight mothers. This would indicate an imbalance in the microbiota of breast milk, as previously documented in regard to gut microbiota in allergic, inflammatory, and obese states $(36,37)$.

Considering our study data together with those from previous publications on the influence of maternal parameters on early and later infant health, the series of clinical demonstrations would 
Table 5. Impact of maternal BMI on microbial composition (prevalence and levels) of breast milk samples during first 6 mo of breastfeeding

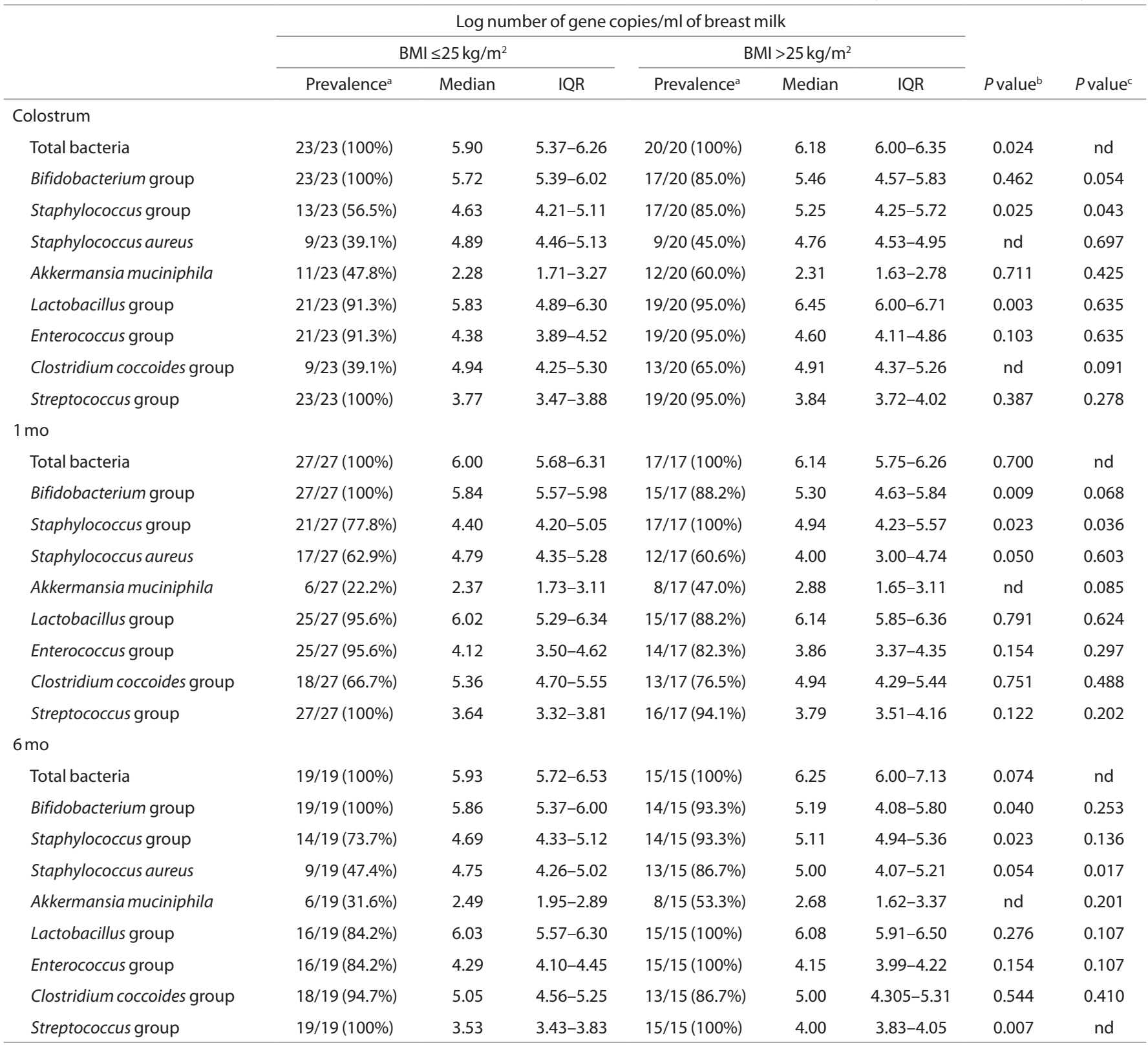

IQR, interquartile range; nd, statistical analysis was not possible due to the number of samples.

aPrevalence reflects the percentage of positive amplifications from total samples analyzed by PCR ( $n=$ number of samples analyzed). ${ }^{b}$ Statistical differences were calculated using Mann-Whitney $U$-test. C Statistical differences in bacterial prevalences between BMI groups (normal and obese) were calculated using $X^{2}$ test ( $2 \times 2$ ).

substantiate how breastfeeding may guide infants through the critical period of transition from immature and inexperienced immune responsiveness to mature barrier functions. Adding to the already well-known merits of breast milk, our study shows the role of microbiota and their relationships with other milk components and underscores the fact that breast feeding is a key factor in the healthy metabolic, immunological, and microbiological programming of the infant's health.

\section{METHODS}

\section{Subjects and Design}

The subjects were selected from an ongoing prospective, randomized, follow-up study of 256 women, starting in April 2002 (NCT00167700; section3, registered through clinicaltrials.gov) (38). Pregnant women were recruited on their first visit to a maternal welfare clinic during the first weeks of gestation. The exclusion criteria of the study were the presence of metabolic or chronic diseases, or other clinical conditions (except allergy, which was not treated as an exclusion criterion). The study complied with the Declaration of Helsinki guidelines as revised in the year 2000. Written informed consent was obtained from the participants, and the study protocol was approved by the ethics committee of the Hospital District of South-West Finland.

For this study, the subjects were selected in accordance with their prepregnancy BMI (calculated as weight $(\mathrm{kg})$ divided by the square of the height $\left(\mathrm{m}^{2}\right)$ ), the practice of early and exclusive breastfeeding, breastfeeding follow-up for $6 \mathrm{mo}$, and availability of breast milk samples. The final sample included a total of 56 subjects: 22 of them overweight $\left(B M I>25 \mathrm{~kg} / \mathrm{m}^{2}\right)$ and 34 normal-weight (BMI $\leq 25 \mathrm{~kg} / \mathrm{m}^{2}$ ) subjects recruited as controls. The subjects were recruited 
Table 6. Impact of maternal weight gain over pregnancy on microbial composition (prevalence and levels) of breast milk samples during first 6 mo of breastfeeding

Log number of gene copies/ml of breast milk

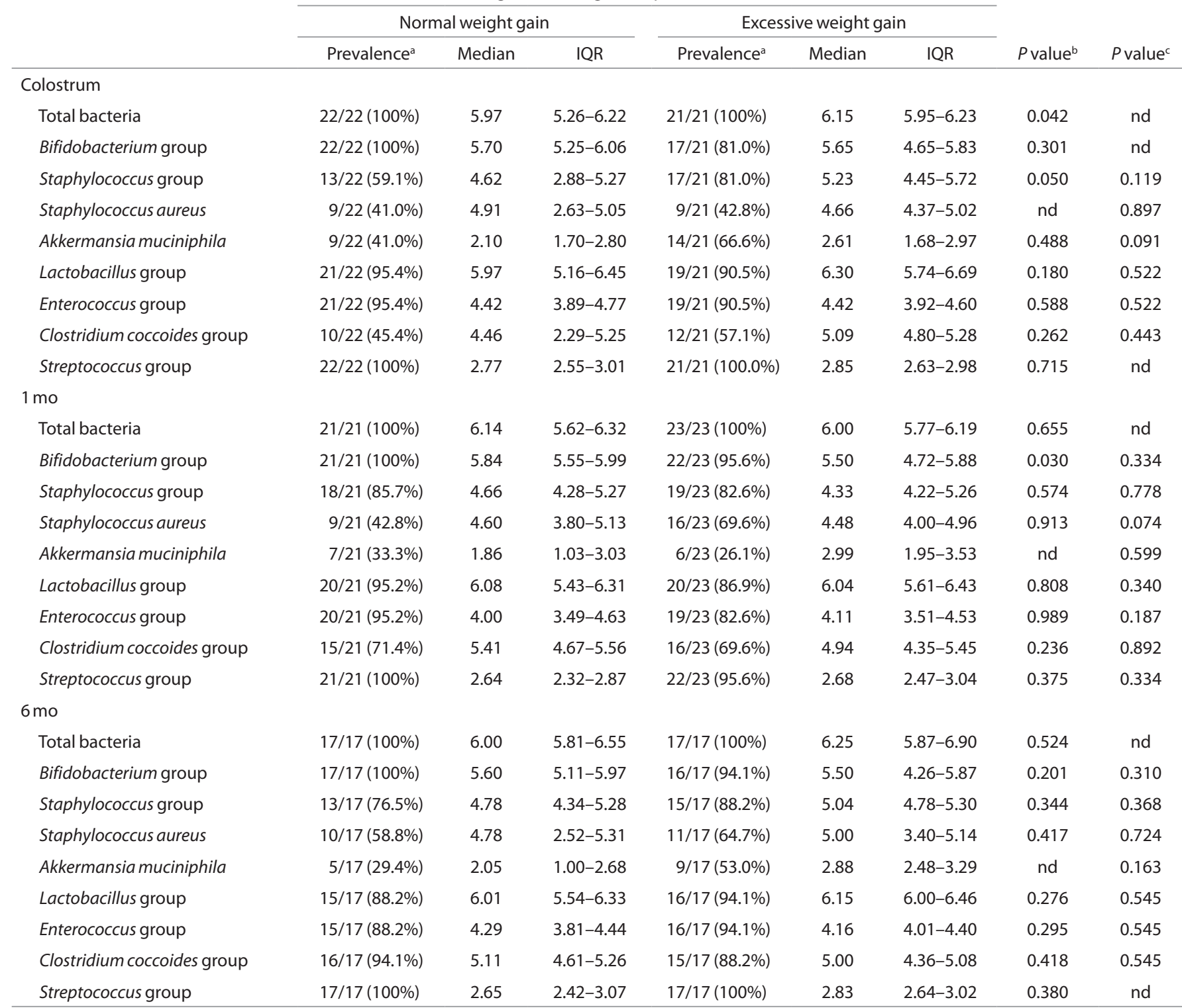

IQR, interquartile range; nd, statistical analysis was not possible due to the number of samples.

aPrevalence reflects the percentage of positive amplifications from total samples analyzed by PCR ( $n=$ number of samples analyzed). ${ }^{\mathrm{b}} \mathrm{Statistical}$ differences were calculated using Mann-Whitney U-test. SStatistical differences in bacterial prevalences between BMI groups (normal and obese) were calculated using $X^{2}$ test $(2 \times 2)$.

in consecutive order, the enrollment of an overweight subject always being followed by the next predefined study number so as to avoid selection bias. Inflammatory and/or infectious processes or conditions such as chorioamnionitis and urinary tract infection were monitored, as was the consumption of anti-inflammatory drugs. Details of the delivery and early infant feeding were collected after birth. After delivery, and at the study visits at 1 and 6 mo thereafter, anthropometric and clinical data of the infants and information on the duration of breastfeeding and infant feeding practices were obtained. "Exclusively breast-fed" was defined as being fed with breast milk alone, without any other liquids or solids; "partially breast-fed" was defined as being breast-fed in addition to being fed complementary foods including milk, infant formula, or semi-solid foods; and "nonbreastfed" was defined as being fed with milk, infant formula, or semi-solid foods, but no breast milk.

To analyze the impact of maternal weight gain during pregnancy on the milk microbiota, weight gain was classified in accordance with the Institute of Medicine (39) recommendations as "normal weight gain," this being $11.5-16.0 \mathrm{~kg}$ and $7.0-11.5 \mathrm{~kg}$, for normal-weight women and overweight women, respectively. Weight gains exceeding the upper values of the recommended ranges $(>16 \mathrm{~kg}$ and $>11.5 \mathrm{~kg}$ for normal-weight and overweight mothers, respectively) were considered as being excessive weight gain.

\section{Breast Milk Samples}

Breast milk samples (colostrum) were collected in the maternity hospital within 24-48 h after delivery, and mature milk samples were collected at two time points, at $1 \mathrm{mo}$ and at $6 \mathrm{mo}$ after delivery, at the homes of the subjects. The subjects were given written instructions regarding standardized collection of samples on the mornings of the study visits; the samples were frozen and stored at $-20^{\circ} \mathrm{C}$ for later analysis. Before sample collection, the breast was cleaned with an iodine swab to minimize the skin bacteria entering the sample. The breast milk sample was collected manually using a sterile milk collection unit, after discarding the 
first few drops. A total of 23 colostrum samples, 27 milk samples at $1 \mathrm{mo}$, and 19 milk samples at 6 mo were available from normal-weight mothers, whereas 20,17 , and 15 samples at these respective time points were available from overweight mothers.

\section{DNA Extraction and Quantitative Real-Time PCR}

The samples of colostrum and of breast milk at 1 and 6 mo after delivery were thawed and centrifuged at $10,000 \mathrm{~g}$ for $10 \mathrm{~min}$ to separate cells and fat from whey, and then concentrated. Thereafter, total DNA was isolated from the pellets using the QIAamp DNA Stool Mini Kit (QIAgen, Hilden, Germany) in accordance with the manufacturer's instructions. The same equipment was also used to extract DNA from pure cultures of the various microorganisms for the purposes of standards. The PCR primers used for the characterization of breast milk microbiota in this study have been previously described (9). They included total bacteria, Bifidobacterium group, BacteroidesPrevotella group, Clostridium coccoides, Staphylococcus group and Staphylococcus aureus, Akkermansia muciniphila, Lactobacillus group, Enterococcus group, and Streptococcus group. These oligonucleotides were purchased from Thermo Electron (Thermo Biosciences, Ulm, Germany). Quantitative PCR amplification and detection were performed using an ABI PRISM 7300-PCR sequence detection system (Applied Biosystems, Foster City, CA). Each reaction mixture of $25 \mu \mathrm{l}$ was composed of SYBR Green PCR Master Mix (Applied Biosystems), $0.5 \mu \mathrm{l}$ of each of the specific primers at a concentration of $0.25 \mu \mathrm{M}$, and $1 \mu \mathrm{l}$ of template DNA. The fluorescent products were detected in the last step of each cycle. A melting-curve analysis was made after amplification to distinguish the targeted PCR product from the nontargeted PCR product. The bacterial concentration in each sample was calculated by comparing the threshold cycle $(\mathrm{Ct})$ values obtained from standard curves. These were created using serial 10 -fold dilution of pure culture-specific DNA fragments corresponding to 10 to $10^{9}$ number of gene copies $/ \mathrm{ml}$.

\section{Determination of Cytokine Concentrations in Breast Milk}

The concentrations of TGF- $\beta 2$ and sCD14 in whey were measured using commercial sandwich enzyme-linked immunosorbent assays specific for these molecules (R\&D Systems Europe, Abingdon, UK). Cytokines IFN- $\gamma$, TNF- $\alpha$, IL-10, IL-6, IL-4, and IL-2 in whey were measured by means of a multiplexed flow cytometric assay, using a commercial human cytokine kit (BD CBA Human Th1/Th2 Cytokine Kit II, cat. no. 551809; BD Immunocytometry Systems, Dendermonde, Belgium). Before analysis, $12 \mathrm{mmol} / \mathrm{l}$ of sodium taurocholate synthesized from cholic acid (Sigma-Aldrich, Basel, Switzerland) was added to an equal volume of milk samples, and these were incubated for $30 \mathrm{~min}$ before being centrifuged at $12,000 \mathrm{~g}$.

\section{Statistical Analyses}

Statistical analyses were carried out using SAS version 9.2. software for Windows (SAS Institute, Cary, NC). For the BMI and weight gain groups, microbial data were expressed as medians with interquartile ranges, and the Mann-Whitney test was used for comparison. Differences in prevalence rates of bacterial groups were established by applying the $\chi^{2}$ test. Univariate associations between two continuous variables were studied using Spearman's correlation.

In addition, associations between cytokines and microbiota were studied using Tobit regression. The cytokine distributions were skewed to the right and were, therefore, logarithmically transformed before parametric analysis. Mixed models for left-censored repeated measures were used to study the association between independent variables and microbiota (40). This method, which involves taking left-censored values into account when estimating parameters, is meant to maximize a full likelihood of distinguishing the contributions of observed measures and left-censored measures to the bacteria load. Time, BMI, weight gain, and cytokine values were the independent variables whose effects on microbiota were studied individually. Ordinary mixed-model repeated-measures analysis was used to study the association between independent variables and cytokines. Time, BMI, weight gain, and cytokine values were the independent variables, whose effects on cytokines were studied individually. The results relating to cytokines and microbes are given as geometric means and, by reason of logarithmic transformation, the group comparisons are given as rate ratios with $95 \%$ CIs. A $P$ value $<0.05$ was considered statistically significant for all tests.

\section{ACKNOWLEDGMENTS}

We thank Jaakko Matomaki for statistical consultation during the data analysis. All authors participated in the preparation of the manuscript.

\section{STATEMENT OF FINANCIAL SUPPORT}

We gratefully acknowledge financial support from the Social Insurance Institution of Finland, the Sigrid Jusélius Foundation, and the Academy of Finland. A "Ramón y Cajal" research contract (RYC-2010-05614) from the Ministry of Science and Innovation, Spain, and grant 069/2010 from Consellería de Sanidad, GVA, Spain (to M.C.C.), are gratefully acknowledged.

Disclosure: None of the authors had any personal or financial conflicts of interest.

\section{REFERENCES}

1. Petherick A. Development: Mother's milk: A rich opportunity. Nature 2010;468:S5-7.

2. Walker A. Breast milk as the gold standard for protective nutrients. J Pediatr 2010;156:2 Suppl:S3-7.

3. LeBouder E, Rey-Nores JE, Raby AC, et al. Modulation of neonatal microbial recognition: TLR-mediated innate immune responses are specifically and differentially modulated by human milk. J Immunol 2006;176:3742-52.

4. Gueimonde M, Laitinen K, Salminen S, Isolauri E. Breast milk: a source of bifidobacteria for infant gut development and maturation? Neonatology 2007;92:64-6.

5. Brandtzaeg P. 'ABC' of mucosal immunology. Nestle Nutr Workshop Ser Pediatr Program 2009;64:23-38; discussion 38-43, 251-7.

6. Innis SM. Metabolic programming of long-term outcomes due to fatty acid nutrition in early life. Matern Child Nutr 2011;7:Suppl 2:112-23.

7. Le Huërou-Luron I, Blat S, Boudry G. Breast- v. formula-feeding: impacts on the digestive tract and immediate and long-term health effects. Nutr Res Rev 2010;23:23-36.

8. Kalliomäki M, Collado MC, Salminen S, Isolauri E. Early differences in fecal microbiota composition in children may predict overweight. Am J Clin Nutr 2008;87:534-8.

9. Collado MC, Isolauri E, Laitinen K, Salminen S. Effect of mother's weight on infant's microbiota acquisition, composition, and activity during early infancy: a prospective follow-up study initiated in early pregnancy. Am J Clin Nutr 2010;92:1023-30.

10. Bäckhed F, Ding H, Wang $T$, et al. The gut microbiota as an environmental factor that regulates fat storage. Proc Natl Acad Sci USA 2004;101:15718-23.

11. Turnbaugh PJ, Ley RE, Mahowald MA, Magrini V, Mardis ER, Gordon JI. An obesity-associated gut microbiome with increased capacity for energy harvest. Nature 2006;444:1027-31.

12. Cani PD, Neyrinck AM, Fava F, et al. Selective increases of bifidobacteria in gut microflora improve high-fat-diet-induced diabetes in mice through a mechanism associated with endotoxaemia. Diabetologia 2007;50:2374-83.

13. Shankar K, Harrell A, Liu X, Gilchrist JM, Ronis MJ, Badger TM. Maternal obesity at conception programs obesity in the offspring. Am J Physiol Regul Integr Comp Physiol 2008;294:R528-38.

14. Picciano MF. Human milk: nutritional aspects of a dynamic food. Biol Neonate 1998;74:84-93.

15. Strobel S. Oral tolerance, systemic immunoregulation, and autoimmunity. Ann N Y Acad Sci 2002;958:47-58.

16. Böttcher MF, Abrahamsson TR, Fredriksson M, Jakobsson T, Björkstén B. Low breast milk TGF-beta2 is induced by Lactobacillus reuteri supplementation and associates with reduced risk of sensitization during infancy. Pediatr Allergy Immunol 2008;19:497-504. 
17. Godlewski MM, Slupecka M, Wolinski J, et al. Into the unknown-the death pathways in the neonatal gut epithelium. J Physiol Pharmacol 2005;56:Suppl 3:7-24.

18. Snijders BE, Stelma FF, Reijmerink NE, et al. CD14 polymorphisms in mother and infant, soluble CD14 in breast milk and atopy development in the infant (KOALA Study). Pediatr Allergy Immunol 2010;21:541-9.

19. Luoto R, Kalliomäki M, Laitinen K, et al. Initial dietary and microbiological environments deviate in normal-weight compared to overweight children at 10 years of age. J Pediatr Gastroenterol Nutr 2011;52:90-5.

20. Böttcher MF, Jenmalm MC, Garofalo RP, Björkstén B. Cytokines in breast milk from allergic and nonallergic mothers. Pediatr Res 2000;47:157-62.

21. Kalliomäki M, Ouwehand A, Arvilommi H, Kero P, Isolauri E. Transforming growth factor-beta in breast milk: a potential regulator of atopic disease at an early age. J Allergy Clin Immunol 1999;104:1251-7.

22. Rautava S, Kalliomäki M, Isolauri E. Probiotics during pregnancy and breast-feeding might confer immunomodulatory protection against atopic disease in the infant. J Allergy Clin Immunol 2002;109:119-21.

23. Rautava S, Arvilommi H, Isolauri E. Specific probiotics in enhancing maturation of IgA responses in formula-fed infants. Pediatr Res 2006;60:221-4.

24. Nermes M, Kantele JM, Atosuo TJ, Salminen S, Isolauri E. Interaction of orally administered Lactobacillus rhamnosus GG with skin and gut microbiota and humoral immunity in infants with atopic dermatitis. Clin Exp Allergy 2011;41:370-7.

25. Youngster I, Kozer E, Lazarovitch Z, Broide E, Goldman M. Probiotics and the immunological response to infant vaccinations: a prospective, placebo controlled pilot study. Arch Dis Child 2011;96:345-9.

26. Matsuda F, Chowdhury MI, Saha A, et al. Evaluation of a probiotics, Bifidobacterium breve BBG-01, for enhancement of immunogenicity of an oral inactivated cholera vaccine and safety: a randomized, double-blind, placebo-controlled trial in Bangladeshi children under 5 years of age. Vaccine 2011;29:1855-8.

27. Perez PF, Doré J, Leclerc M, et al. Bacterial imprinting of the neonatal immune system: lessons from maternal cells? Pediatrics 2007;119:e724-32.

28. Donnet-Hughes A, Perez PF, Doré J, et al. Potential role of the intestinal microbiota of the mother in neonatal immune education. Proc Nutr Soc 2010;69:407-15.
29. Rescigno M, Rotta G, Valzasina B, Ricciardi-Castagnoli P. Dendritic cells shuttle microbes across gut epithelial monolayers. Immunobiology 2001;204:572-81.

30. Qutaishat SS, Stemper ME, Spencer SK, et al. Transmission of Salmonella enterica serotype typhimurium DT104 to infants through mother's breast milk. Pediatrics 2003;111(6 Pt 1):1442-6.

31. Collado MC, Derrien M, Isolauri E, de Vos WM, Salminen S. Intestinal integrity and Akkermansia muciniphila, a mucin-degrading member of the intestinal microbiota present in infants, adults, and the elderly. Appl Environ Microbiol 2007;73:7767-70.

32. Derrien M, van Passel MW, van de Bovenkamp JH, Schipper RG, de Vos WM, Dekker J. Mucin-bacterial interactions in the human oral cavity and digestive tract. Gut Microbes 2010;1:254-68.

33. van Passel MW, Kant R, Zoetendal EG, et al. The genome of Akkermansia muciniphila, a dedicated intestinal mucin degrader, and its use in exploring intestinal metagenomes. PLoS ONE 2011;6:e16876.

34. Collado MC, Isolauri E, Laitinen K, Salminen S. Distinct composition of gut microbiota during pregnancy in overweight and normal-weight women. Am J Clin Nutr 2008;88:894-9.

35. Aaltonen J, Ojala T, Laitinen K, Poussa T, Ozanne S, Isolauri E. Impact of maternal diet during pregnancy and breastfeeding on infant metabolic programming: a prospective randomized controlled study. Eur J Clin Nutr 2011;65:10-9.

36. Delzenne NM, Cani PD. Interaction between obesity and the gut microbiota: relevance in nutrition. Annu Rev Nutr 2011;31:15-31.

37. Ley RE. Obesity and the human microbiome. Curr Opin Gastroenterol 2010;26:5-11.

38. Laitinen K, Poussa T, Isolauri E. Probiotics and dietary counselling contribute to glucose regulation during and after pregnancy: a randomised controlled trial. Br J Nutr 2009;101:1679-87.

39. Nutrition during pregnancy, weight gain and nutrient supplements. Report of the Subcommittee on Nutritional Status and Weight Gain During Pregnancy. Subcommittee on Dietary Intake and Nutrient Supplements During Pregnancy and Lactation, Food and Nutrition Board. Institute of Medicine. Washington, DC: National Academy Press, 1990, 1-233.

40. Thiébaut R, Jacqmin-Gadda H. Mixed models for longitudinal left-censored repeated measures. Comput Methods Programs Biomed 2004;74: 255-60. 